\title{
A Model for the Invasion and Spread of Rhizomania in the United Kingdom: Implications for Disease Control Strategies
}

\author{
Adrian J. Stacey, James E. Truscott, Michael J. C. Asher, and Christopher A. Gilligan
}

Epidemiology and Modelling Group, Department of Plant Sciences, University of Cambridge, Downing Street, Cambridge CB2 3EA, UK. Current address of J. E. Truscott: Department of Infectious Disease Epidemiology, Faculty of Medicine, Imperial College, Norfolk Place, London W2 1PG, UK.

Accepted for publication 27 August 2003.

\begin{abstract}
Stacey, A. J., Truscott, J. E., Asher, M. J. C., and Gilligan, C. A. 2004. A model for the invasion and spread of rhizomania in the United Kingdom: Implications for disease control strategies. Phytopathology 94:209-215.

Rhizomania disease of sugar beet represents a major economic threat to the sugar industry in the United Kingdom. Here we use the UK rhizomania epidemic as an exemplar of a range of highly infectious spatially heterogeneous diseases. Using a spatially explicit stochastic model, we investigated the efficacy of a spectrum of possible control strategies, both locally reactive and national in character. These include the use of novel

show that strictly local responses, including a containment policy similar to that initially implemented in the United Kingdom in response to the disease, are largely ineffective in slowing the spread because they fail to match the natural scale of the epidemic. Larger spatial-scale processes are considerably more successful. We conclude that epidemics have intrinsic temporal and spatial scales that must be matched by any control strategy if it is to be both effective and efficient. We have generated probability distributions for the proportion of farms symptomatic. Over the course of the epidemic, such distributions develop a bimodality that we hypothesize to correspond to the matching of spatial heterogeneity in the susceptible population to the intrinsic scales of the epidemic.
\end{abstract} cultivars of beet with different responses to infection, changes in cultivation practice, and reactive containment policies at the farm scale. We
Additional keywords: resistance, tolerance, variability.
The invasion of diseases into susceptible populations is a recurring and contemporary problem, with recent examples including foot and mouth disease in the United Kingdom $(5,10)$ and citrus canker in South America and Florida (6,7). Analysis, control and prediction of such diseases are often confounded by the presence of asymptomatic hosts which are nevertheless capable of transmitting infection (17). Such an asymptomatic phase is a property of many contagious diseases (1); however, it is particularly prolonged for a range of soilborne plant pathogens. Such soilborne pathogens are exemplified by rhizomania disease of sugar beet. In this paper, we model and assess control strategies for the developing epidemic of rhizomania disease in the United Kingdom. In contrast with the systems originally modeled by Van der Plank (17), this epidemic is particularly complex because the asymptomatic phase is coupled with disease dynamics and control measures that have strong spatial and stochastic components. Because these aspects of rhizomania typify those of many diseases, the conclusions have broad applicability for a range of pathogens.

Rhizomania was first detected in the United Kingdom in 1987 (Fig. 1). After a slow initial phase between 1987 and 1990, the number of outbreaks per year has started to increase rapidly. This is of particular concern because the disease can have a severe impact on yield and persists in the soil almost indefinitely (2). Forecasting the severity and spatial pattern of the disease and predicting the response of the epidemic to control strategies is therefore very important.

The causative agent of the disease is the Beet necrotic yellow vein virus (BNYVV). The virus is transmitted through the vector

Corresponding author: J. E. Truscott; E-mail address: j.truscott@imperial.ac.uk

Publication no. P-2003-1124-02R

(c) 2004 The American Phytopathological Society
Polymyxa betae, a soilborne protist that infects the fibrous roots of sugar beet plants. The virus is able to persist in the overwintering spores, or cystosori, of the parasite. The parasite itself causes no significant harm to the host but introduces the virus to the root system where it rapidly multiplies (2). The infection causes a proliferation of lateral roots. These roots harbor the viruliferous resting spores and those that remain in the soil after harvest add to the inoculum present for the next crop. The amplification of inoculum in the soil resulting from the growth and harvesting of a particular cultivar of beet is termed the amplification rate for that cultivar. The details of this amplification process and its modeling are described below. Infection is largely confined to the roots of the host and symptoms above ground only occur when the level of infestation exceeds a threshold level. One important consequence is that the infestation of a field may not become apparent until several beet crops have been grown. We define the inoculum concentration at which symptoms appear as the threshold level for any given cultivar. The combination of the threshold for symptoms and crop rotation with periods generally greater than 3 years results in disease remaining undetected in beet-growing fields for more than a decade. As a result, soil infestation is much more extensive than symptoms indicate.

Because the infectious agent is soilborne, spread of the disease on a scale larger than that of the root system of a single host can only be due to the movement of soil. Although P. betae has a freeliving zoospore stage in its life cycle, these zoospores have a range of only a few centimeters. On dry and sandy soils, the spread of soil can occur through wind blow, but in general the movement of agricultural equipment is the dominant agent of dispersal at the field, farm, and regional scales $(8,12)$. Movement of agricultural equipment carrying significant quantities of soil takes place primarily at the time of harvest; consequently, the temporal and spatial dynamics of the disease are separate. During a growing season, the disease does not spread, and while the inoculum is 
dispersing it cannot amplify. The details of the dynamics of dispersal and its modeling are described below.

There are a number of possible strategies for restricting the spread of an epidemic. When outbreaks of rhizomania were first observed, a local containment policy was implemented by the UK Ministry of Agriculture, Fisheries and Food (MAFF), now the Department for the Environment, Food and Rural Affairs (DEFRA). The policy requires that farms showing rhizomania symptoms should cease future beet cultivation in the infested fields and disinfect machinery that has passed through them. We examine the long-term consequences of this policy on the epidemic. At present, much effort is going into the development of resistant cultivars as an alternative method of disease control. We look at the effect of different inoculum amplification rates and asymptomatic threshold levels on the control of the epidemic. We also assess the influence of changes in cultivation practice, such as isolation of fields (improved farm hygiene) and reduction of farm-to-farm contact rates, on disease progress. Finally, we examine the effect of the scale of spatial heterogeneity in the distribution of beet farms on the reliability of control strategies.

\section{MATERIALS AND METHODS}

We developed a model for the amplification and dispersal of BNYVV. The model describes the states of all farms and fields involved in beet production. The locations of the farms and their relationship to each other are modeled explicitly. Amplification of inoculum within a crop is treated as a deterministic process, while all scales of the transmission process are stochastic, governed by probability distributions extracted from outbreak data. To examine the effect of control strategies, the model was encapsulated in a national-scale simulator for rhizomania in the United Kingdom. The simulator allowed parameters of the model to be adjusted during the course of a run to represent the effect of changing control strategy. From the stochastic output of the simulator, statistics for the numbers of infested, symptomatic, and disease-free farms and fields were generated.

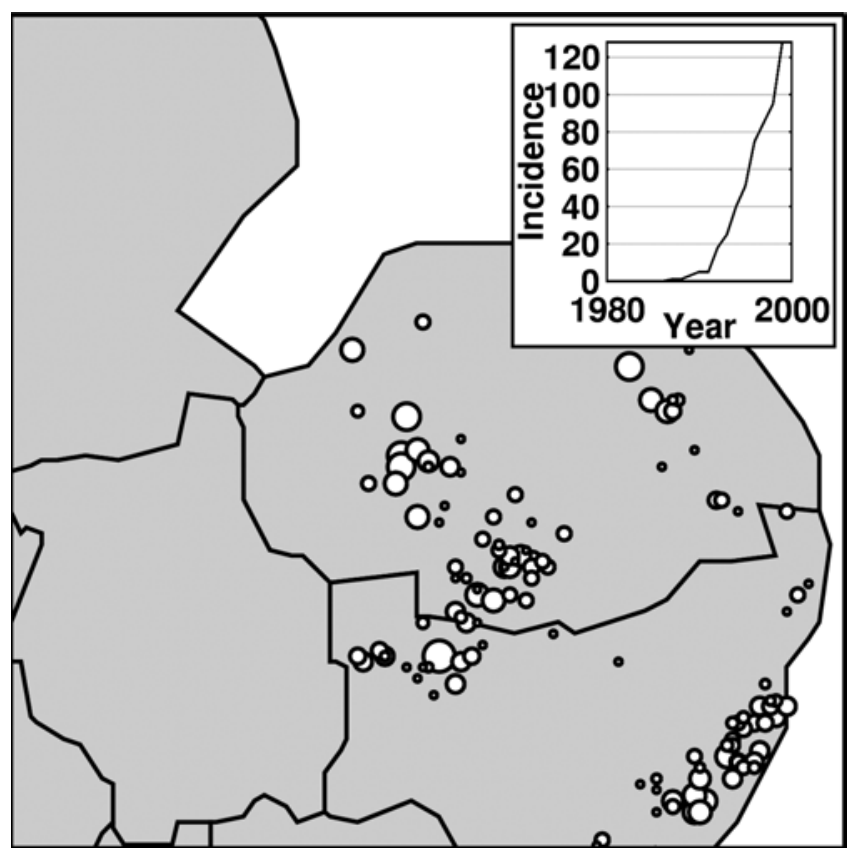

Fig. 1. History of the rhizomania in the United Kingdom up to 2000. The circles in the main diagram indicate the location of rhizomania outbreaks. Circle diameters indicate year of outbreak. The largest circles denotes the year 1987, the smallest 2000. The inset shows the cumulative number of outbreaks as a function of year.
Using the simulator, we examined the effects of a variety of control strategies on the progress of the epidemic in the United Kingdom. In particular, we investigated the effect of implementing the use of cultivars of beet with different responses to the disease, locally implemented containment policies in response to the appearance of symptoms, and several possible variations in beet cultivation practice. These strategies are described in detail below. We first introduce the disease model.

The model. Progress of the disease is modeled in terms of the dynamics of the soilborne viruliferous inoculum that attacks the host crop. The development of the epidemic is divided into two alternating phases: amplification and dispersal. These two depend on each other only to the extent that the end of each phase determines the initial conditions for the next. The amplification phase comprises only temporal evolution and is confined to the growing season of the sugar beet crop. Within this period, some root contact between plants is possible, but the short length scale of transmission between roots makes its effect on spatial redistribution of inoculum negligible in comparison with transfer on machinery. The dispersal phase involves the movement of infectious material in its resting spore form. Hence, during this phase, no amplification can occur. Stochasticity is introduced into the spatial part of the model within the mechanisms governing how much and to where inoculum is distributed.

Temporal dynamics. The amplification of inoculum in the soil is the result of the infection of the root system by the vector $P$. betae, carrying BNYVV, the increase of the virus load within the fibrous roots, and the return of the roots to the bulk of the soil at harvest. The root system is vulnerable to attack in two ways, defined as primary and secondary infection. In primary infection, the soilborne inoculum units infect roots that pass close to them. In secondary infection, motile propagules are generated from infected roots. These motile propagules then cause new infections in neighboring roots.

A detailed temporal model of this process is developed in Webb et al. (18). However, analysis of the model $(14,15)$ reveals it to have two main modes of behavior, depending on the relative strength of the secondary infection cycle. In the primary infection mode, the ability of the fungus to infect the root system by secondary infection is critically weaker than the ability of the plant to produce roots that become resistant. In effect, the root system outgrows the fungus and is largely unharmed. In the secondary infection mode, the secondary cycle is sufficiently strong for the positive feedback mechanism of secondary infection to dominate. This allows the fungus to colonize a large proportion of the root system. Hence, the plant becomes heavily infected with rhizomania. The temperature dependence of the parameters of the system means that this qualitative change of behavior is seen as a sharp temperature-dependent change in the apparent force of infection of the fungus (3), with little infection below a critical temperature threshold and rapid amplification above that threshold.

For the purposes of fitting to data and use within the full model, it is convenient to develop an analytically tractable submodel that characterizes these two modes. This model describes the amplification of inoculum over a season while encapsulating the main features of the full temporal model described in Truscott et al. (14):

$$
\begin{aligned}
\text { Total roots: } & \mathrm{d} n / \mathrm{d} t=r(1-n) \\
\text { Susceptible roots: } & \mathrm{d} s / \mathrm{d} t=r(1-n)-\lambda(T) M_{i} s-m s \\
\text { New inoculum: } & \mathrm{d} M / \mathrm{d} t=Q \lambda(T) M_{i} s
\end{aligned}
$$

Here, $n$ represents the total root population, $s$ the susceptible population, and $M$ the new inoculum. The parameters $r, m$, and $Q$ represent root growth rate, the inverse of the susceptible period for the roots, and the amplification of inoculum within infected 
root tissue, respectively. The parameter $\lambda(t)$ is the force of infection per unit inoculum initially present in the vicinity of the plant, where the initial inoculum is given by $M_{i}$. To characterize the switch from primary to secondary infection mode, it is assumed that the infection process is either "on" or "off" with the switch at temperature, $T_{c}$; that is,

$$
\lambda(T)= \begin{cases}\lambda_{m} & T \geq T_{c} \\ 0 & T<T_{c}\end{cases}
$$

This is justified by the extreme difference in force of infection between the two modes. It is convenient to assume that there exists a critical time in the season, $t_{c}$, at which the mean temperature exceeds the critical temperature, $T_{c}$. In a season, the system runs from $t=0$ to $t_{f}$, which is harvest. Before $t_{c}$, no new inoculum is produced, so effectively we integrate the system through $\left[t_{c}, t_{f}\right]$ to give

$$
s\left(t_{c}\right)=s(0) \exp \left(-m \times t_{c}\right)+\frac{r\left(1-n_{0}\right)}{m-r}\left[\left(\exp \left(-r \times t_{c}\right)-\exp \left(-m \times t_{c}\right)\right]\right.
$$

For the period $\left[t_{c}, t_{f}\right]$, we have input into the inoculum class, $M$,

$$
M\left(t_{f}\right)-M\left(t_{c}\right)=Q \lambda_{m} M_{i}\left(A\left\{1-\exp \left[-Z\left(t_{f}-t_{c}\right)\right]\right\}+B\left\{1-\exp \left[-r\left(t_{f}-t_{c}\right)\right]\right\}\right)
$$

where

$$
\begin{gathered}
A=\frac{1}{Z}\left(s\left(t_{c}\right)-\frac{r\left(1-n_{0}\right)}{Z-r} \exp \left(-r \times t_{c}\right)\right) \\
B=\frac{1-n_{0}}{Z-r} \exp \left(-r \times t_{c}\right) \\
Z=\lambda_{m} M_{i}+m
\end{gathered}
$$

The crop is considered symptomatic if, at the end of the season, $M>M_{c}$, the threshold inoculum level. Beet cultivar influences the amplification process in two ways: the amplification rate of a cultivar modulates the parameter $Q$ and the critical threshold modifies the parameter $M_{c}$. The above functions for inoculum generation were fitted to data from inoculum amplification experiments $(3,16)$ in order to estimate the parameters $r, m$, and $Q$ for conventional cultivars. Within the model, the variables governing total and susceptible root densities were left nondimensional, representing the proportion of the total root density achievable. The parameters were estimated using the method of least squares (Table 1). Data concerning regional variation in soil type and climate were included in the model through their effect on the rate of warming of the soil. Soil-type data (supplied by DEFRA) were input into the simulator with a granularity of $1 \mathrm{~km}$.

Spatial dynamics. The transmission of viruliferous inoculum occurs on a range of scales: within a field, between fields on a farm, and between farms on a regional scale. We assume that the dominant mode of transport is through the movement of agricultural machinery $(8,12)$. Inoculum is moved around a field primarily during the harvest of the infected crop. The movement of machinery to other fields on the same farm and to other farms sharing that machinery distributes the inoculum on larger scales.

Spread within a field. We model the effect of moving machinery on any single unit of inoculum using a probability distribution function. This distribution describes the probability that an inoculum particle moves a given distance due to cultivation. The effect of cultivation on the distribution of inoculum in the whole field is calculated by convoluting the spatial pattern of inoculum in the field with this probability distribution. This approach follows that of Brain and Marshall (4) and Marshall and Brain (11), who showed that the movement of objects in the soil by cultivation can be described by a probability distribution for the distance moved. The distribution is effectively exponential in form,

$$
p(r) \mathrm{d} r=\left\{\begin{array}{cc}
k \exp (-k \times r) & r \geq 0 \\
0 & r<0
\end{array}\right.
$$

where $1 / k$ is the mean displacement of moved inoculum and $r$ is distance.

The importance of this process within a field lies not with the displacement of inoculum but in its diluting effect. When a focus of infection is cultivated, inoculum is redistributed and the peak concentration is reduced. This reduction delays the time at which the peak inoculum concentration reaches the threshold level and causes visible symptoms. A diluting factor can be calculated from the redistribution kernel to represent the effect of cultivation on peak inoculum concentration (13). This diluting effect is conveniently summarized in the model by representing the inoculum in any individual field by two linked quantities, the total inoculum present, which determines the force of infection from that field (described below), and the peak inoculum concentration, which defines when a field becomes symptomatic. The total inoculum present is subject to amplification only, whereas the peak is subject to both amplification and dilution. The parameter $k$ was estimated from aerial photographs of symptomatic fields, and the analysis of distribution patterns is given in Truscott and Gilligan (13).

Spread within and between farms. Spread between fields on a farm and between farms is assumed to occur by essentially the same mechanism, namely, the movement of machinery at the relevant scale. We define two parameters, $p_{i}$ and $p_{b}$, which represent the Poisson mean of the number of potentially infectious journeys made by machinery within and between farms, respectively. A majority of transmission is assumed to take place directly after harvest. At this time, the viruliferous inoculum arising on a farm is equal to the total amount of inoculum in beet-growing fields on that farm at the end of a season. At the start of any particular journey, a small proportion of the transmissible inoculum is assumed to be picked up by the machinery. This proportion is assumed to be Poisson-distributed in consideration of the repeated "sampling" of soil on the infested fields by the machinery during cultivation.

For each journey, a target farm is selected. For within-farm transmission, this target is always the farm from which the inoculum was picked up; for between-farm transmission, the target is chosen at random from among the contacts of this farm. In the latter case, each potential contact is chosen with the same probability. Inoculum is then distributed randomly among fields on the target farm. For simplicity, we assume that the number of fields visited by the machinery is Poisson-distributed. There is circumstantial evidence for this from patterns of outbreaks on farms early in the rhizomania epidemic (data for outbreaks on individual farms from DEFRA).

TABLE 1. Parameter values as fitted to outbreak data from 1984 to 2000

\begin{tabular}{lccc}
\hline Description & Symbol & Value & Units $^{\mathrm{a}}$ \\
\hline Amplification & & & \\
$\quad \begin{array}{l}\text { Growth rate } \\
\text { Reciprocal of susceptible }\end{array}$ & $r$ & 0.1 & day $^{-1}$ \\
$\quad$ period for root & $m$ & 0.07 & $\mathrm{day}^{-1}$ \\
$\quad \begin{array}{l}\text { Force of infection } \\
\text { Amplification }\end{array}$ & $\lambda_{m}$ & 0.84 & ${\text { (days IU } / 100 \mathrm{~g})^{-1}}^{(\text {days IU } / 100 \mathrm{~g})^{-1}}$ \\
$\quad$ Standard threshold & $Q$ & 2.37 & - \\
Transmission & $M_{c}$ & 0.066 & \\
$\quad$ Mean dispersal in field & $k$ & 0.2 & $\mathrm{~m}^{-1}$ \\
Mean number of contacts & $c_{a}$ & 16 & - \\
Contact radius & $R_{c}$ & 10 & $\mathrm{~km}^{-}$ \\
Mean number of farm visits & $p_{b}$ & 4 & - \\
$\quad$ Mean number of field visits & $p_{i}$ & 0.3 & - \\
Mean external infection rate & $e_{x}$ & 0.5 & year $^{-1}$ \\
\hline
\end{tabular}

${ }^{\mathrm{a}} \mathrm{IU}$ stands for inoculum units. 
To construct the network of farm contacts through which transmission takes place, we assume that each farm has an optimum number of contacts with which it would share machinery, drawn from a Poisson distribution (mean, $c_{n}=16$ ) at the start of each simulation run. Contacts are then formed randomly between farms within an economically viable radius of each other, $R_{c}$, until no further contacts can be found. Contracts are formed subject to the constraint that no farm exceeds its maximum number of contacts and that each contact is reciprocal. In addition, we assume that spread of inoculum from outside the United Kingdom occurs according to a Poisson distribution with a mean, $e_{x}$, of 0.5 infections per year. This corresponds to the rate of the appearance of new foci in the first 10 years of the epidemic.

Clearly the transmission of inoculum within and between farms is also dependent upon the nature of any local or national containment policies in place and also on the responses of the growers themselves to the presence of the disease. These aspects are discussed below.

The spatial model was fitted to data for the positions and times of appearance of symptomatic farms in the United Kingdom between the start of the epidemic and 2000, supplied by DEFRA. This was achieved by first defining a number of characteristic measures on the data, $\chi_{i}(i=0,1,2)$, namely, the number of symptomatic farms each year, the mean distance of the symptomatic farms from the epicenter of infestation, and the quotient of the mean distance of symptomatic farms from the epicenter and the time since initial symptom appearance. Simulated data were then generated from the model for particular sets of parameter values, and the values of the measures were calculated from these data. Probability distributions, $p_{i}\left(\chi_{i}\right)$, were constructed for the values of each of the measures, $\chi_{i}$. We generated the equivalent values of the measures, $\mu_{i}$, from the outbreak data (Fig. 1). The likelihood of any particular parameter set is then $\Pi p_{i}\left(\mu_{i}\right)$. In this way, a likelihood surface was built up from which the most probable parameter combination was chosen.

We tested the full model by assessing its ability to reproduce the patterns of rhizomania spread that have been observed since the start of the rhizomania epidemic until the present day. The numbers of occurrences of newly symptomatic farms and fields from year to year were accurately predicted by the model. In addition, the model reproduced the spatial distribution of the epidemic as it evolved. The model remained accurate from 2000 to the present day (that is, for dates that were not in the range of data used to parameterize the model). This corroborates the mechanisms used for the spatial redistribution of inoculum as described previously. In addition, we used the full model to predict the number of outbreaks up until 2090. A large number of iterations (runs of the model from the start of the epidemic until 2090) were used to determine not only the expected behavior of the epidemic but also the probability distribution of the predictions.

Evaluation of control strategies. We evaluated a range of control strategies by encapsulating the model described previously in a simulator that adjusts the parameters of the model in response to the details of the strategy being tested.

We first tested the effect of different cultivars on the progress of the epidemic. Two physiological traits were selected, amplification rate and inoculum symptom threshold. The control strategy used allowed the epidemic to develop naturally up to the year 2000, at which point the new cultivar was introduced throughout the United Kingdom. We allowed the amplification rate and symptom thresholds to vary over a range of several orders of magnitude from their values in a standard commercial cultivar and examined the effect on the proportion of symptomatic and infected farms by 2050.

We examined the effect of MAFF's containment policy as a measure to prevent the spread of disease from infested farms. Under this policy, all subsequent cultivation of sugar beet was forbidden on any field that had previously shown symptoms of rhizomania or had tested positive for BNYVV in an enzymelinked immunosorbent assay (ELISA) test. Hygiene on the farm was also tightened, with machinery and clothing being disinfected after contact with the symptomatic field. On a national scale, import restrictions were put in place to prevent introduction of new inoculum from abroad.

Within the model, the prohibition on growing beet in a symptomatic field and enhanced hygiene around that field have the effect of removing the field as a source of inoculum for other fields on the farm and other connected farms. This can be inferred from the assumption that transmission events occur directly after the harvest of the beet crop. In this paper, we have not addressed import restrictions, because current data are insufficient to quantify their effects. To test the containment policy within the simulator, we compared the spatial pattern of the epidemic in 2050 for the case in which containment was in place up to 2000 with the case in which containment remained in place until 2050. Relatively few iterations are required for spatial maps because the process of producing such a map implies a degree of pseudoreplication across regions of space within any given iteration (Fig. 2).

In addition, we examined what impact changes in cultivation practice can have on the spread of rhizomania. We also tested the influence of the effect of limiting the redistribution of infectious material from field to field on an infected farm regardless of whether symptoms are expressed.

\section{RESULTS}

Figure 2A illustrates the asymptomatic margin of rhizomania disease (the proportion of farms that are infested but not symptomatic), and Figure 2B illustrates the spatial pattern of disease outbreaks predicted by the model for the year 2050. The predicted incidence of rhizomania is spatially highly heterogeneous due to the combined effects of (i) variation in soil type and temperature, which lead to differential rates of amplification and hence spread, (ii) the pattern of initial infestation, which occurred predominantly in the east of the country, and (iii) the distribution of sugar beet growers, which are sparse in the center of the country. In addition, there is a stochastic component to the model which accounts for some of the nuances of differences between the maps for different treatments (Fig. 2C to E).

One of the most important features of the epidemic from the point of view of restricting the spread of the disease is this asymptomatic margin. From Figure 2A, it is clear that the farms that are infested but not symptomatic form a thin region ahead of the expanding symptomatic front (map B), approximately $20 \mathrm{~km}$ wide. Because random ELISA testing is laborious, expensive, and inefficient, it is generally the presence of symptoms that triggers detection of the disease. This asymptomatic region is therefore normally invisible; its width represents how far ahead of the visible front the epidemic has already progressed. It results from the spread of viruliferous inoculum from farms at the edge of the disease front that are not yet showing symptoms.

Figure 3 shows a sequence of probability distributions for the proportion of farms symptomatic for different points in the epidemic. The mean of the distributions increases approximately linearly with time, suggesting that, averaged over many realizations, the disease is effectively a steadily advancing wavefront through the country. The variance of the distribution increases with time, showing that the speed of the advancing wave has a probability distribution of its own. Figure 3C shows a bimodal distribution for the proportion of symptomatic farms in 2090. This is caused by the variability in the local densities of beetgrowing farms. In some iterations, by chance, the disease crosses regions of low farm density (such as the Pennines) and reaches regions of higher farm density and spreads rapidly. In other iterations, the low density region provides a barrier to further spread of the disease. The bimodality thus results from an interaction 
between the characteristic spatial scale which enables continued spread of the epidemic and heterogeneity in the distribution of hosts.

The effectiveness of local containment in restricting the spread of the disease can be judged by comparing maps $\mathrm{B}$ and $\mathrm{C}$ in Figure 2. Map B shows the distribution of symptomatic farms in 2050 under a containment policy implement in 1984 and revoked in 2000. Map C shows the effect of continuing the containment policy up to 2050. Clearly, the aspects of the containment policy considered here have a negligible effect. The containment policy fails because the movement of viruliferous inoculum is not restricted until symptoms appear on a farm. Furthermore, the policy predominantly affects inoculum spread only from symptomatic fields. By the time a field becomes symptomatic, however, the disease has already spread throughout the asymptomatic margin. The containment policy is implemented on each farm too late to be effective, because it involves a reaction to the appearance of symptoms (termed a reactive policy). The same argument would apply to any other field or farm-scale reactive policies.

Another possible means of control of the disease is through a general change in practice for sugar beet cultivation, regardless of the disease status of the farm. Map D in Figure 2 shows the effect of successfully preventing the transmission of infectious inoculum from field to field within a farm. By comparing this to the standard epidemic progress (Fig. 2, map B), it is clear that this has very little controlling effect on the epidemic. We denote farmto-farm transmission as primary infestation, and field-to-field transmission within a farm as secondary infestation. Secondary
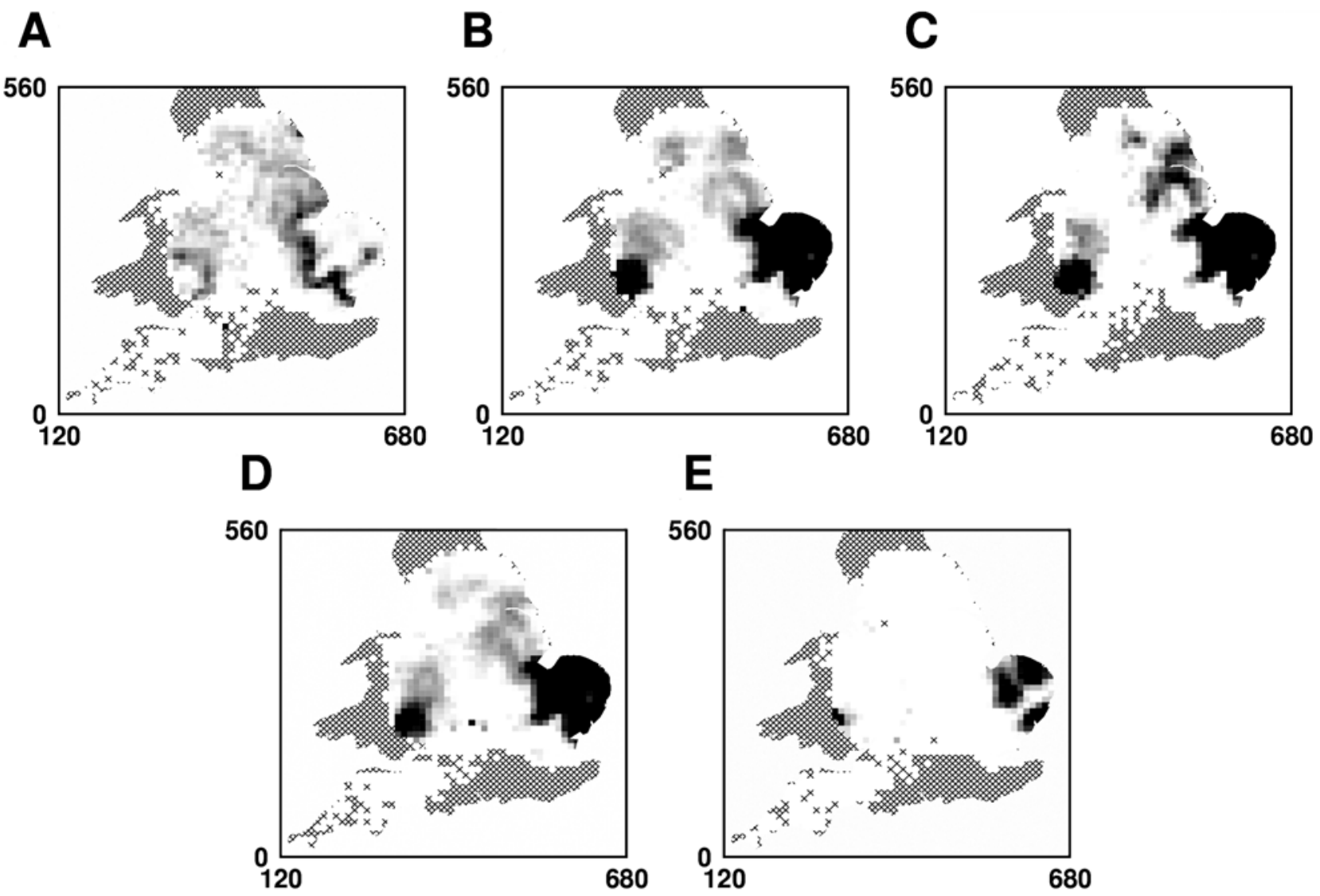

Fig. 2. All maps represent the predicted state of the epidemic in the year 2050. Maps A and B show the spatial distribution of asymptomatic (infested) and symptomatic farms, respectively, under standard parameter values (Table 1). The remaining maps show the distribution of symptomatics as follows: C, containment implemented from 2000, D, restriction of within-farm transmission from the year 2000, and $\mathbf{E}$, restriction of between-farm transmission to $10 \%$ of its standard level from the year 2000. Darker shades indicate that a larger proportion of farms in a region are symptomatic. Cross-hatching indicates regions where beet is not grown.

\section{A 2010}

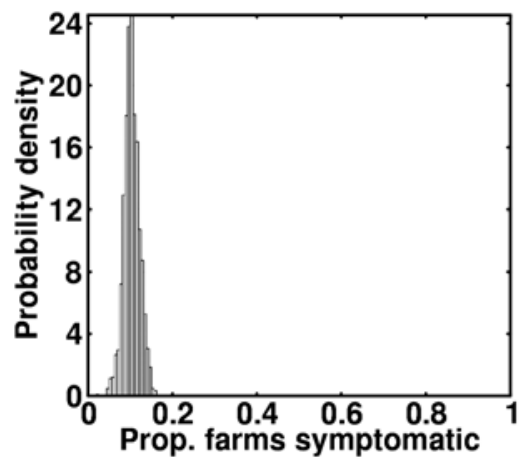

B 2050

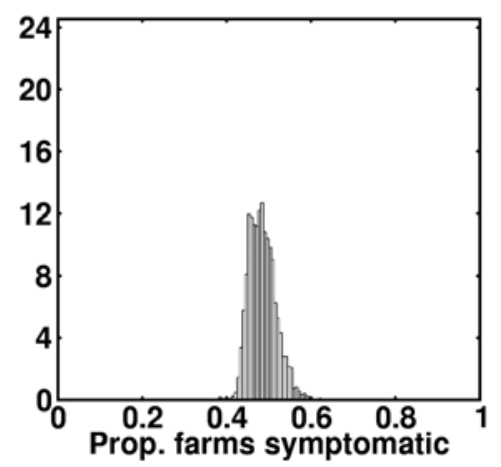

C 2090

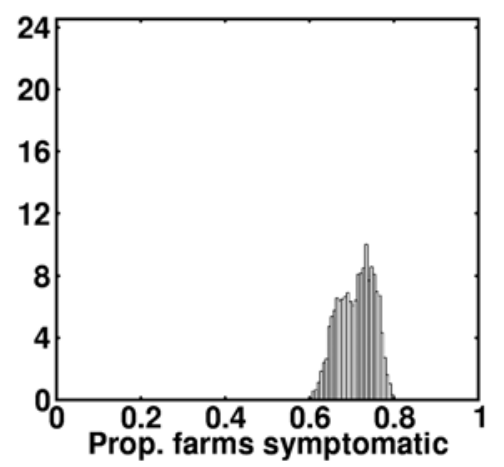

Fig. 3. Probability distributions for the number of symptomatic farms in the years A, 2010, B, 2050, and C, 2090 under standard fitted parameter values (Table 1). 
infestation increases the probability that a farm will transmit infection. However, the time-lag for secondary infestation to occur is sufficiently large for the primary infestation process to dominate in terms of affecting the large-scale spread of the epidemic. By the time secondary infestation has occurred, the disease front has already moved on.

As an alternative strategy, we investigated the effect of changing the number of infectious journeys made between farms. Map E of Figure 2 shows the density of symptomatic farms in 2050 after the mean number of farm-to-farm visits has been reduced to $10 \%$ of its fitted value in the year 2000 . This clearly leads to a large reduction in the number and extent of symptomatic farms by 2050 in comparison with the standard fitted parameter values. The effectiveness of these strategies can be interpreted in terms of the scale at which they act. The key to the spread of the disease nationally is its ability to spread between farms. Transmission of inoculum has to be controlled at the same scale.

The use of certain novel cultivars can be effective against the spread of rhizomania, as shown in Figure 4. Graphs A and B show the fraction of infested and symptomatic farms in 2050 as a function of the threshold level and amplification rate of the cultivars, respectively. Containment was maintained throughout the evolution of the system. The proportion of symptomatic farms has a monotonic dependence on the symptom threshold of the host plant. As the threshold becomes large, the fraction of symptomatic farms falls. Not enough generations of beet are being grown for the inoculum to reach symptomatic levels. The proportion of infested farms is unchanged as the threshold increases, showing that the epidemic has not been restricted, only hidden. For small thresholds, both the number of symptomatic and infested farms decline. The epidemic is being controlled to some extent by this measure. When the threshold is small, symptoms appear earlier in the series of beet crops, eventually appearing in the first crop after infestation for extreme values. As a result, the reactive containment policy comes into play sufficiently early to limit the spread of the disease effectively.

Figure 4B shows the influence of amplification rate on the fractions of symptomatic and infested farms. As amplification rate increases, the quantity of inoculum transmitted at each contact increases, and hence the number of farms infested also increases. In addition, the threshold for symptoms is reached more quickly and fewer generations of beet are grown before containment comes into force. However, this secondary effect is insufficient to halt the epidemic. Spread is stopped simply by exhausting the susceptible population. For low rates of amplification, the background infested level includes those farms that were infested in the initial period and their contacts. The background symptomatic level comprises those farms that become symptomatic within the initial phase. It is instructive to compare this to the low threshold limit in graph A (Fig. 4A). This comprises farms infested in the initial period, their contacts, and those infested since the new cultivar was introduced. Since in this case symptoms appear in the first season, the difference between these two values represents the spread brought about by the transmission after the initial harvest.

\section{DISCUSSION}

A key conclusion to be drawn from the results is that there exist key time and space scales for the epidemic that must be matched for any successful control strategy. The time scale of the epidemic is defined by the delay between the infestation of a field and the appearance of symptoms, 10 to 15 years in this case. For a reactive control strategy, the space scale is the distance the disease can be transmitted over this time. From Figure $2 \mathrm{~A}$ we can see that this is approximately $20 \mathrm{~km}$. It is clear that any reactive strategy responding to the appearance of symptoms and acting on the farm scale will therefore fail, as illustrated in Figure $2 \mathrm{C}$. The epidemic has already passed before measures take effect. Modeling of the foot and mouth epidemic in cattle (9) leads to a similar evaluation of local reactive containment strategies: the scale of local containment needs to match the spatial scale of the epidemic to be effective. For global strategies, such as changes in farming practice, strategies aimed at limiting transmission must still be targeted at the correct scale. However, in this case, the scale is that of the dominant transmission process. The stringent hygiene policy put in place globally in Figure 2D fails because it is primarily farmto-farm transmission that is responsible for progress of the disease front. Tackling the farm-to-farm scale, in contrast (Fig. 2, map E), has a marked effect on epidemic extent and severity.

Global changes in cultivar attempt to control disease by limiting the quantity of inoculum (amplification rate) or its impact (threshold). Increasing the disease threshold can never halt the spread of inoculum and can only hide its continuing spread. In the short term, as shown in Figure 4A, this can appear to ameliorate the epidemic. However, it will continue to spread and will eventually appear unless complete tolerance can be achieved in the crop. Surprisingly, a very low threshold reduces the time scale for

\section{B Amplification}

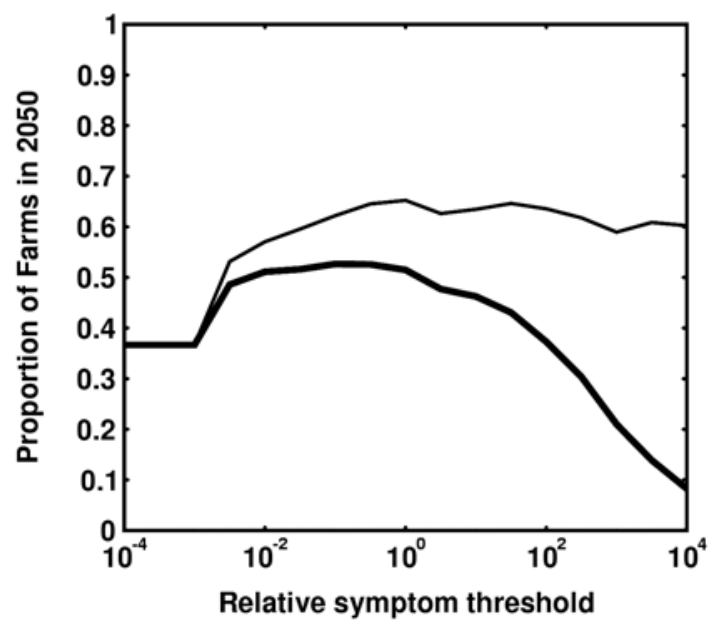

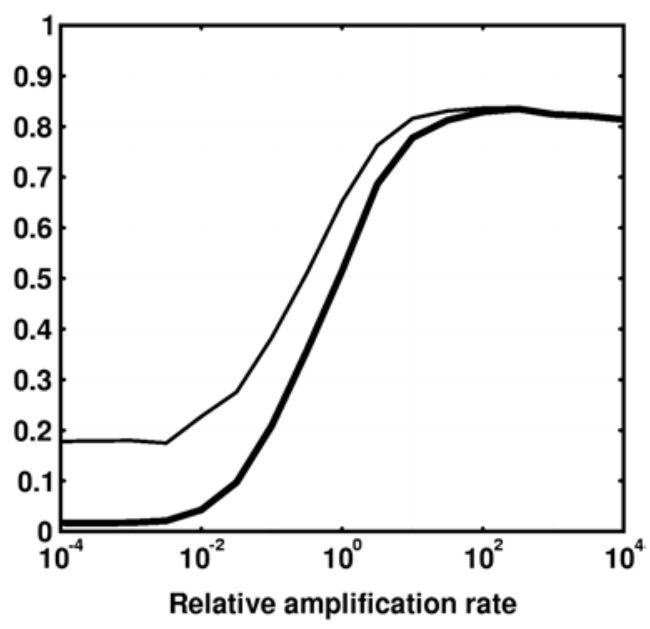

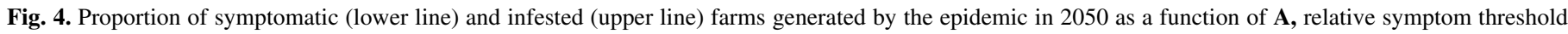

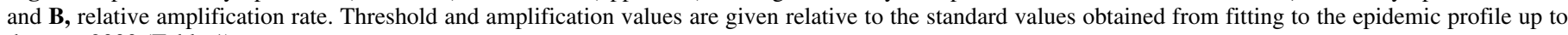
the year 2000 (Table 1). 
the disease development. This reduces the spatial scale of the epidemic to such an extent that it is commensurate with that of the MAFF containment strategy. A global reduction in amplification rate is clearly an effective control simply by quenching the production of viruliferous inoculum. However, it should be noted that the fraction of symptomatic farms has at best a log dependence on amplification rate. Large reductions $(\approx 1 \%$ of the fitted values) are needed to control the epidemic effectively.

Novel cultivars of beet may differ from conventional cultivars in terms of both amplification rate and symptom threshold. We consider now a novel cultivar that exhibits both an increase in threshold and a decrease in amplification rate; in practice, cultivar trials would tend to favor such a cultivar. Such a cultivar would produce an immediate benefit in suppressing symptom development and inoculum amplification. However, the time between infestation and symptoms being observed would be increased by both the change in threshold and amplification rate. Hence, the asymptomatic period for the disease, and the spatial scale at which control would have to be applied, would be particularly large for such a cultivar. For rhizomania, this would have negligible impact on the success of control; the MAFF policy, as described here, is ineffective even for the current spatial scale of the disease. However, for a different disease-control policy combination in which the control policy initially matched the spatial scale of the epidemic, and hence, restricted the spread of the disease, the introduction of such a novel cultivar has the potential to increase the spatial scale of the epidemic beyond that of the control policy. The initially beneficial effect of the introduction of such a semiresistant cultivar would be followed by the 'escape' of the disease from control and larger scale infestation than if the cultivar had not been used.

The probability distribution functions shown in Figure 3 raise an interesting point about the interaction of the scales of the spatial distribution mechanisms of the epidemic and the scales of heterogeneity in the environment through which it is spreading. The bimodality exhibited in Figure $3 \mathrm{C}$ results from variability in whether the epidemic crosses regions of low farm density. In these low-density regions, the separation of susceptible farms is of the same order as the mean farm-to-farm transmission distance. On the eastern side of the United Kingdom, through which the epidemic is spread (Fig. 3A and B), farms growing sugar beet are more closely spaced. Hence, the largely Gaussian distributions seen in graphs A and B reflect the stochasticity of the underlying epidemic, whereas graph $\mathrm{C}$ is modulated by the interaction of the heterogeneity of the environment and the space scale of the epidemic (Fig. 3).

The interaction between rhizomania and the control strategies used to counter its spread in the United Kingdom represents an important contemporary example of a broad spectrum of hostpathogen control combinations. This paper suggests that one of the essential aspects of such combinations to consider for a successful strategy is the match between the space and time scales of the epidemic and those of the control strategy. Further work will aim at identifying approximations for estimating the space and time scales of the epidemic and control strategies, enabling the efficacy of control strategies to be assessed without recourse to simulation of a whole system.

\section{ACKNOWLEDGMENTS}

This work was funded by the Ministry of Agriculture, Fisheries and Food (now the Department for the Environment, Food, and Rural Affairs) in the UK, the British Sugar Beet Corporation, and the Biotechnology and Biological Sciences Research Council. We thank the two anonymous referees and L. L. Kinkel for detailed and helpful comments on a draft of the paper.

\section{LITERATURE CITED}

1. Anderson, R. M., and May, R. M. 1992. Infectious Diseases of Humans: Dynamics and Control. Oxford University Press, Oxford.

2. Asher, M. J. C. 1993. Rhizomania. Pages 313-346 in: The Sugar Beet Crop: Science Into Practice. D. Cooke and R. Scott, eds. Chapman and Hall, London.

3. Blunt, S. J., Asher, M. J. C., and Gilligan, C. A. 1992. Infection of sugar beet by Polymyxa betae in relation to soil temperature. Plant Pathol. 41:257-267.

4. Brain, P., and Marshall, E. 1996. Estimating cultivation effects on weed seed dispersal using Fourier transforms. Aspects Appl. Biol. 46:173-179.

5. Ferguson, N. M., Donnelly, C. A., and Anderson, R. M. 2001. Transmission intensity and impact of control policies on the foot and mouth epidemic in Great Britain. Nature 413:542-548.

6. Gottwald, T. R., Hughes, G., Graham, J. H., Sun, X., and Riley, T. 2001. The citrus canker epidemic in Florida: The scientific basis of regulatory eradication policy for an invasive species. Phytopathology 91:30-34.

7. Gottwald, T. R., Timmer, L. W., and McGuire, R. G. 1989. Analysis of citrus canker in nurseries in Argentina. Phytopathology 79:1276-1283.

8. Heijbroek, W. 1988. Dissemination of rhizomania by soil, beet seeds and stable manure. Neth. J. Plant Pathol. 94:9-15.

9. Keeling, M. J., Woodhouse, M. E., May, R. M., Davies, G., and Grenfell, B. T. 2003. Modelling vaccination strategies against foot-and-mouth disease. Nature 421:136-142.

10. Keeling, M. J., Woodhouse, M. E., Shaw, D. J., and Matthews, L. 2001. Dynamics of the 2001 UK foot and mouth epidemic: Stochastic dispersal in a dynamic landscape. Science 294:813-817.

11. Marshall, E. J. P., and Brain, P. 1999. The horizontal movement of seeds in arable soil by different soil cultivation methods. J. Appl. Ecol. 36: 443-454.

12. Richard-Molard, M. 1985. Rhizomania: A world-wide danger to sugar beet. Span 28:92-94.

13. Truscott, J. E., and Gilligan, C. A. 2001. The effect of cultivation on the size, shape and persistence of disease patches in fields. Proc. Nat. Acad. Sci. 98:7128-7133.

14. Truscott, J. E., Webb, C. R., and Gilligan, C. A. 1997. Asymptotic analysis of an epidemic model with primary and secondary infection. Bull. Math. Biol. 59:1101-1123.

15. Truscott, J. E., Webb, C. R., and Gilligan, C. A. 2000. Quantitative analysis and model simplification of an epidemic model with primary and secondary infection. Bull. Math. Biol. 62:366-393.

16. Tuitert, G., and Hofmeester, Y. 1992. Epidemiology of beet necrotic yellow vein virus in sugar beet at different initial inoculum levels in the presence or absence of irrigation: Dynamics of inoculum. Neth. J. Plant Pathol. 98:343-360.

17. Van der Plank, J. E. 1963. Plant Diseases: Epidemics and Control. Academic Press, New York.

18. Webb, C. R., Gilligan, C. A., and Asher, M. J. C. 1999. A model for the temporal build-up of Polymyxa betae. Phytopathology 89:30-38. 